STRUCTURAL SCIENCE CRYSTAL ENGINEERING MATERIALS

ISSN 2052-5206

\section{Lab in a DAC - high-pressure crystallography as a powerful tool to study chemical interactions and chemical reactions}

\author{
Elena V. Boldyreva*
}

Boreskov Institute of Catalysis SB RAS, Lavrentieva, 5, Novosibirsk, 630090, Russian Federation, and Novosibirsk State University, Pirogova, 2, Novosibirsk, 630090, Russian Federation. *Correspondence e-mail: eboldyreva@catalysis.ru

It is my great pleasure to write a scientific commentary on a recent review by Andrzej Katrusiak (Katrusiak, 2019). Time goes fast, and I have hardly noticed the two decades after the IUCr Congress in Glasgow in 1999, where I first met Andrzej in person (Fig. 1) and we could exchange our experience in high-pressure single-crystal diffraction studies of intermolecular interactions in the crystals of organic compounds. I had just entered this field in which Andrzej has been already active as one of the pioneers (Katrusiak, 1991). There were not so many people at that time who were interested in this topic.

Since that time both the hardware [diffractometers, diamond anvil cells (DACs)] and the software have developed to such an extent that a single-crystal high-pressure X-ray diffraction experiment can provide data of a completeness and precision that enables structure solution and refinement for unknown phases of great complexity, so that even an electron charge density analysis becomes possible (Casati et al., 2017). Detailed studies of chemical bonds, intermolecular interactions, subtle details of structural transformations of organic crystals under extreme conditions became mainstream in chemical crystallography (Boldyreva, 2018). Research on hydrostatic compression can also be very helpful in order to understand the structural transformations at ambient pressure (Zakharov \& Boldyreva, 2019). The sample sealed in the DAC can be compressed and heated to the chosen starting pressure and temperature and then processed by changing their values. In these changing conditions one can investigate the stability regions of a compound and its phase transitions, and this information can be used for drawing the pressure-temperature ( $\mathrm{p}-\mathrm{T}$ ) phase diagrams, in a similar way as done in high-pressure thermal analysis and calorimetry. The extreme conditions in the DAC can be applied for performing chemical reactions which otherwise would require catalysts, would proceed with much smaller yields or would not proceed at all.

The recent review by Katrusiak (2019) gives a personal overview of this exciting field. When DACs were first designed and manufactured 60 years ago, the main interest of researchers was focused on studying minerals and inorganic compounds. The progress of solving structures of organic compounds at high pressures can be illustrated by an increase in the number of crystal structures deposited in the Cambridge Structural Database (Groom et al., 2016), reaching 2500 entries by the end of 2019. One relatively short article cannot cover the whole variety of high-pressure crystallographic experiments, the new designs of high-pressure equipment, computer programs for theoretical computations for high-pressure structures and for the interpretation of high-pressure data. Therefore, this research perspectives article focuses on selected aspects most relevant to crystal engineering and the research results changing the understanding of concepts in materials chemistry, undoubtedly biased by this author's personal interests. Still, the author and his research group have performed such extensive and variable research at high pressures throughout the decades that even being based mainly on a personal experience, the paper gives a balanced and multi-faceted overview of the field.

In the early years of high-pressure X-ray diffraction studies a researcher was excited to find a new high-pressure polymorph and to solve its structure. Nowadays, the challenge is to find some universal laws and rules of high-pressure transformations. The paper by Katrusiak (2019) suggests some of such possible rules. In particular, the author discusses the role of pressure-transmitting fluids in the compression of solids, the reasons why 


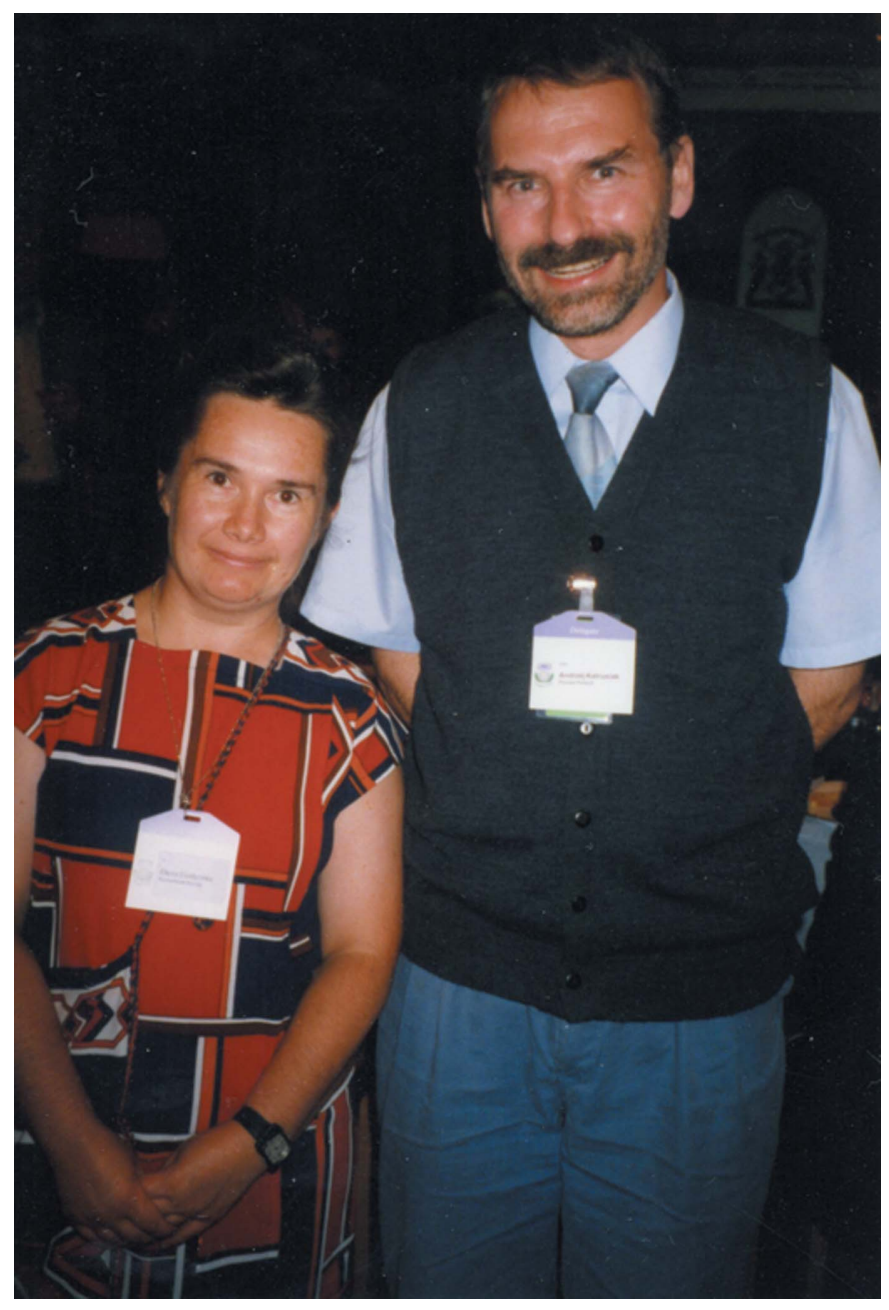

Figure 1

The author of this commentary with Andrzej Katrusiak in 1999 at the 18th IUCr Congress in Glasgow.

compression on cooling and on increasing pressure can be drastically different, and why the rule of inverse effects of temperature and pressure that has been formulated for inorganic solids often does not hold for organic compounds, why the inverse relation rule does not apply to the symmetry relations between low-pressure and high-pressure phases. The problem of predicting if a multi-component crystalline phase will form or, on the contrary, decompose on hydrostatic compression is discussed using solvates, hydrates, salts and cocrystals as examples. The increase in coordination numbers in the high-pressure phases as compared to the ambient-pressure counterparts is discussed and compared with an alternative possibility of increasing the crystal density by filling in the voids by guest molecules of penetrating fluids. Structural effects induced by high pressure in crystals can be so strong that they cannot be regarded solely as a physical transformation, as they change the chemical character of substances, even before the chemical reaction actually takes place. For example, the bond lengths, molecular conformation, coordination and interactions can be reversibly changed and correlated with the properties of materials under study. Thus, high pressure can induce changes in the chemical bonding similar to those characteristic for chemical reactions. This can considerably facilitate the research on functional materials in the search for their structure-properties relationships. Examples of such transformations are also given.

In general, this paper by Katrusiak represents a significant step forward in attracting attention to the new possibilities that high-pressure crystallography opens for chemical crystallography and the research of molecular materials. I would recommend it as a very informative and stimulating read to a broad community.

\section{References}

Boldyreva, E. V. (2018). In Understanding Intermolecular Interactions in the Solid State - Approaches and Techniques, Monographs in Supramolecular Chemistry No. 26, edited by D. Chopra, pp. 32-97. UK: Royal Society of Chemistry.

Casati, N., Genoni, A., Meyer, B., Krawczuk, A. \& Macchi, P. (2017). Acta Cryst. B73, 584-597.

Groom, C. R., Bruno, I. J., Lightfoot, M. P. \& Ward, S. C. (2016). Acta Cryst. B72, 171-179.

Katrusiak, A. (1991). Cryst. Res. Technol. 26, 523-531.

Katrusiak, A. (2019). Acta Cryst. B75, 918-926.

Zakharov, B. A. \& Boldyreva, E. V. (2019). CrystEngComm, 21, 1022. 